\title{
Repair Method for Complete High Ulnar Nerve Injury Based on Nerve Magnified Regeneration
}

This article was published in the following Dove Press journal:

Therapeutics and Clinical Risk Management

\author{
Wenquan Ding ${ }^{1} * *$ \\ Xueyuan $\mathrm{Li}^{1, *}$ \\ Jiadong Pan' \\ Peixun Zhang ${ }^{2}$ \\ Shanqing Yin' \\ Xianting Zhou' \\ Junjie $\mathrm{Li}^{\prime}$ \\ Liping Wang $\mathbb{I D}^{1,3}$ \\ Xin Wang' \\ Jianghui Dong $\mathbb{D}^{1,3}$ \\ 'Department of Hand Surgery, \\ Department of Plastic Reconstructive \\ Surgery, Ningbo No. 6 Hospital, Ningbo \\ 315040, People's Republic of China; \\ ${ }^{2}$ Department of Trauma Orthopedics, \\ Peking University People's Hospital, \\ Beijing 100044, People's Republic of \\ China; ${ }^{3}$ School of Pharmacy and Medical \\ Sciences, and UniSA Cancer Research \\ Institute, University of South Australia, \\ Adelaide, SA 500I, Australia
}

*These authors contributed equally to this work
Correspondence: Xin Wang

Department of Hand Surgery,

Department of Plastic Reconstructive

Surgery, Ningbo No.6 Hospital, Ningbo

315040, People's Republic of China

$\mathrm{Tel} / \mathrm{Fax}+86574-87996193$

Email dr.wangxin@hotmail.com

Jianghui Dong

School of Pharmacy and Medical Sciences,

University of South Australia, Adelaide SA

500I, Australia

Tel +6I8 $830227 \mid 5$

Fax +6I8 83021087

Email Jianghui.dong@mymail.unisa.edu.au
Purpose: Complete high ulnar nerve injury can cause serious sequelae, including residual sensation and loss of movement and especially dysfunction of the intrinsic muscles of the hand. As a solution to treat complete high ulnar nerve injury, we proposed a new repair method for ulnar nerve injury based on nerve-magnified regeneration.

Methods: Twenty-two patients with complete division of the ulnar nerve at a high level who were treated from May 2013 to December 2016 were divided into two groups. The proposed repair method for complete high ulnar nerve injury was performed in group I (11 patients), while the traditional repair method, ie, repair of the original injury site of the ulnar nerve, was used in group II (11 patients).

Results: The results showed no significant difference in the mean sensory scores assigned by the Highet-Zachary scheme (the Highet Scale) between the two groups. The mean Highet Scale score of muscle strength for the first dorsal interosseus muscle was significantly better in group I than that in group II ( $p=0.010)$. In group I, 10 of 11 patients were graded as M4 or M5. Grip strength, pinch strength, and the Disabilities of the Arm, Shoulder, and Hand (DASH) score were significantly better in group I than those in group II ( $\mathrm{p}<0.01)$.

Conclusion: Therefore, this method for complete high ulnar nerve injury based on nervemagnified regeneration can shorten the path of motor nerve regeneration, effectively reduce atrophy of the intrinsic muscles of the hand, and provide better hand function.

Keywords: nerve magnified regeneration, ulnar nerve injury, nerve repair, peripheral nervous system

\section{Introduction}

Complete high ulnar nerve injury usually causes severe sequelae, such as residual sensation, loss of movement, and "claw hand" deformity, and affects fine manipulation of the hand. ${ }^{1,2}$ After repair of the ulnar nerve, the nerve regeneration rate is approximately $1-2 \mathrm{~mm} / \mathrm{d},{ }^{3}$ and substantial time is required for regeneration to reach the intrinsic muscles of the hand. The proximal muscle strength of the limbs that perform gross movements is mostly restored after a long recovery, while the strength of the intrinsic muscles for fine movements is poorly recovered. ${ }^{4}$ Therefore, a nerve repair method for complete high ulnar nerve injury that can reduce atrophy of the intrinsic muscles of the hand and achieve better hand function is urgently needed.

To reduce the impact of the sequelae of high ulnar nerve injury and improve hand function, in addition to repairing the ulnar nerve at the injured site, surgeons must perform surgery in the acute phase (within the time window of acute injury) and the late phase. Although functional reconstruction can improve the "claw hand" deformity, ${ }^{5}$ reconstructing the complex fine movements of the intrinsic muscles of 
the hand remains difficult. Sensory nerve transfer includes transfer of the palmar cutaneous branch of the median nerve to the superficial branch of the ulnar nerve ${ }^{6}$ and endto-side anastomosis of the superficial branch of the ulnar nerve to the third common digital nerves. ${ }^{7}$ Recovery above $\mathrm{S} 3+$ can be achieved after nerve transfer. ${ }^{7}$

Animal experiments have confirmed that two types of nerve-magnified regeneration exist. One type involves a smaller proximal nerve dominating a larger distal nerve, ${ }^{8}$ ie, a proximal axon can dominate up to three or four distal axons by nerve-magnified regeneration. Jiang research group of Peking University verified nerve-magnified regeneration through animal experiments in the rhesus monkey. ${ }^{9}$ Another work demonstrated that recovery of function after nerve endto-side anastomosis is also an example of nerve-magnified regeneration. ${ }^{10}$ Together, nerve-magnified regeneration theory provides a theoretical basis for treating complete high ulnar nerve injury.

The slow nerve regeneration takes a long time to reach the intrinsic muscles of the hand, causing atrophy of these muscles. Shortening the path of motor nerve regeneration is one method to solve this problem. Motor nerve transfer is an effective method to shorten the regenerative path and restore innervation of the intrinsic muscles of the hand. The transfer method involves transposing the distal anterior interosseous nerve to the deep branch of the ulnar nerve at the wrist level. ${ }^{6,11}$ The nerve branch to the pronator quadratus muscle at the wrist plays an important role at the transfer site; this small nerve branch derives from the branch of the anterior interosseous nerve of the median nerve and has few axons compared to the deep branch of the ulnar nerve. ${ }^{12}$ In addition to the branch of the pronator quadratus muscle, the terminal branch of the anterior interosseous nerve is a small sensory nerve ${ }^{13}$ that does not play a role in nerve transfer. If nerve-magnified regeneration is achieved, then the anterior interosseous nerve can reinnervate the intrinsic muscles of the hand via the transposed nerve, thus solving the clinical problem of treating complete high ulnar nerve injury.

Therefore, based on nerve-magnified regeneration, the current work proposed a new repair method for complete high ulnar nerve injury. It includes three aims: 1) to verify nerve-magnified regeneration in a clinical investigation; 2) to explore a repair method for complete high ulnar nerve injury that involves shortening the path of motor nerve regeneration based on nerve-magnified regeneration; and 3) to verify the effectiveness of the repair method for complete high ulnar nerve injury through follow-up assessments.

\section{Materials and Methods}

This study was carried out in accordance with the recommendations of the Ethics Committee of the Ningbo No. 6 Hospital with written informed consent from all subjects. All subjects gave written informed consent in accordance with the Declaration of Helsinki. The protocol was approved by the Ethics Committee of the Ningbo No. 6 Hospital.

\section{Repair Method for Complete High Ulnar Nerve Injury}

The repair method for high ulnar nerve injury includes two steps: the injured ulnar nerve is repaired at the injured site, and then the branch of the pronator quadratus muscle is transposed to the deep branch of the ulnar nerve at the wrist level (Figure 1).

Firstly, the repair method for the injured part of the ulnar nerve was dependent on the presence of a nerve defect. In cases without a nerve defect, nerve fascicular suture can be performed using 9-0 non-invasive sutures under a microscope. In cases with a nerve defect, the autologous nerve (harvested from the autologous sural nerve) or allogeneic nerve grafting can be used to bridge the nerve gap (Figure 1).

Secondly, the nerve branch to the pronator quadratus muscle was transposed to the deep branch of the ulnar nerve at the wrist level. The deep branch of the ulnar nerve was retrogradely, non-invasively separated and anastomosed to the nerve branch to the pronator quadratus muscle. End-to-side anastomosis can be performed if little or no tension is present at the anastomosis site. End-to-end anastomosis can be performed if high tension is present at the anastomosis site. The detailed procedure is described as follows:

First, a longitudinal incision was made in the distal volar forearm to separate subcutaneous tissue and expose the ulnar nerve. The deep and the superficial branches of the ulnar nerve can be visualized at the distal ulnar nerve. The branches were separated retrogradely and noninvasively. Finally, the forearm muscle and tendon groups were retracted to the radial side to expose the pronator quadratus muscle.

Second, the distal anterior interosseous nerve can be visualized at the proximal pronator quadratus muscle. Several branches derive from the distal anterior interosseous nerve to innervate the pronator quadratus muscle. The terminal branch of the anterior interosseous nerve passes through the pronator quadratus muscle 


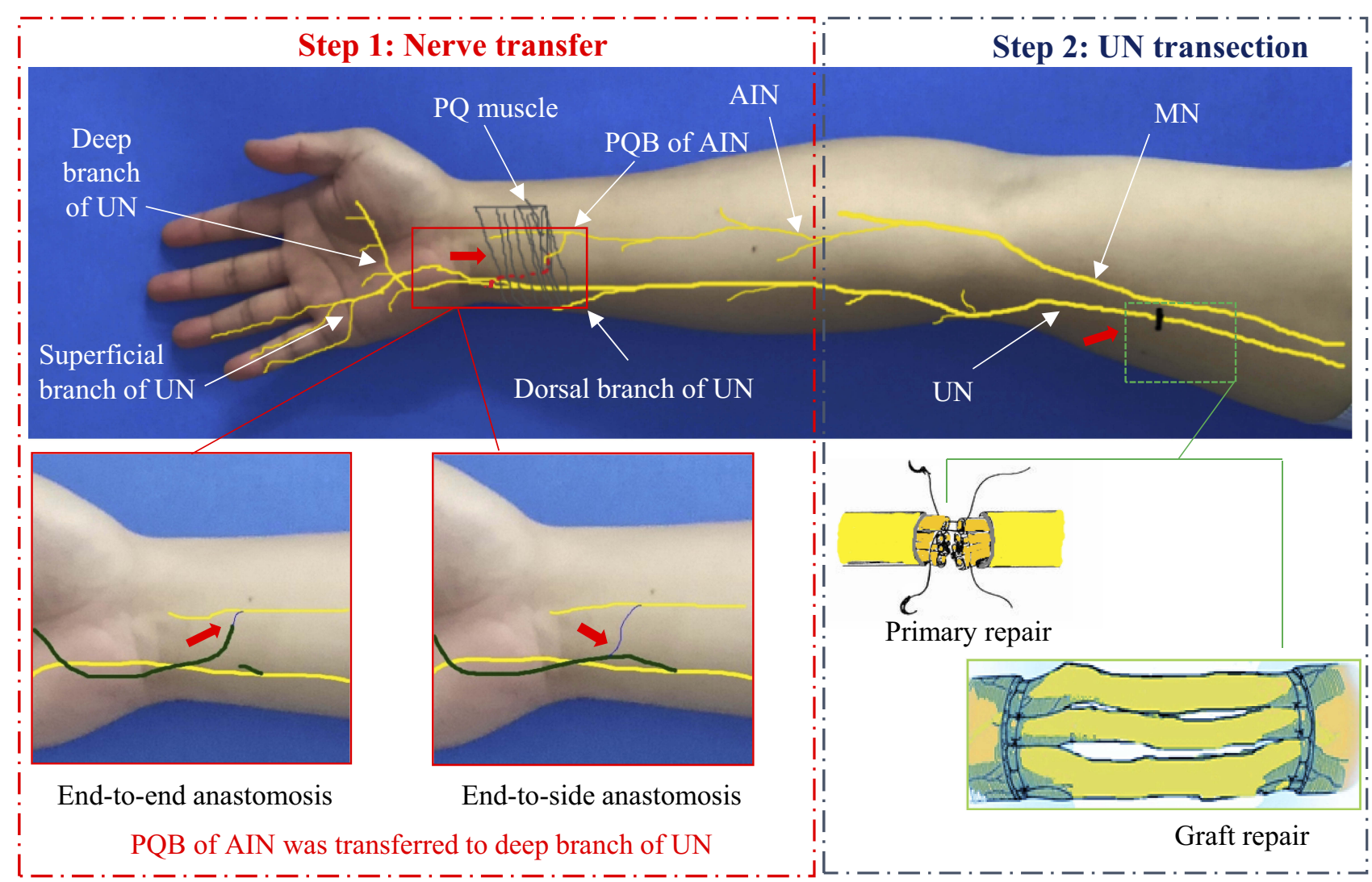

Figure I The injured ulnar nerve is repaired at the injured site (primary or graft repair), and then the branch of the pronator quadratus muscle is transposed to the deep branch of the ulnar nerve at the wrist level (via end-to-end or end-to-side anastomosis). Red arrows indicate where nerve repair is being performed. Abbreviations: MN, median nerve; UN, ulnar nerve; AIN, anterior interosseous nerve; PQ, pronator quadratus; $\mathrm{PQB}$, pronator quadratus branch.

and extends distally. Next, the branch to the pronator quadratus muscle was cut at the farthest distal end of the muscle (when necessary, the terminal branch of the anterior interosseous nerve can be divided). Finally, the free end of the separated branch to the pronator quadratus muscle was pulled to the site of the deep branch of the ulnar nerve.

The tension between the distal branch of the pronator quadratus muscle and the deep branch of the ulnar nerve was evaluated. If no tension was observed, then a 9-0 noninvasive suture was used for end-to-side anastomosis under a microscope (end: distal branch of the pronator quadratus muscle; side: lateral membrane opening of the ulnar nerve deep branch). If tension was observed, then the proximal deep branch of the ulnar nerve was divided, and a 9-0 non-invasive suture was used for end-to-end anastomosis under a microscope.

Routine haemostasis was performed, a negative pressure drainage tube was placed, the skin was closed and dressed with a bandage, and the arm was immobilized using a plaster cast with slight flexion of the wrist.

\section{Repair of Complete High Ulnar Nerve Injury}

\section{Surgical Subjects}

From May 2013 to December 2016, 22 patients with complete high ulnar nerve injury at the Ningbo NO.6 Hospital (Ningbo, China) were included in the work according to the inclusion and exclusion criteria. The inclusion criterion was the presence of complete high ulnar nerve injury, ie, ulnar nerve injury proximal to the upper third of the forearm. The exclusion criteria were age greater than 60 years, peripheral nervous system lesions, fractures, tendon or muscle damage of the ipsilateral hand, radial or median nerve injuries, or old ulnar nerve injuries ( $>2$ months).

According to the repair method for the ulnar nerve, the patients were divided into two groups (group I and group II, Table 1). In group I, 11 patients underwent the proposed repair procedure. In group II (the control group), 11 patients underwent a repair procedure for the injured part of the ulnar nerve without transfer of the anterior interosseous nerve branch of the pronator quadratus muscle to the deep branch of the ulnar nerve. 
Table I Characteristics of Patients with Complete High Ulnar Nerve Injury and Repair

\begin{tabular}{|l|l|l|l|l|l|}
\hline Group & Sex & Age Mean (Range) & Injury Site of Ulnar Nerve & \multicolumn{2}{|l|}{ Repair Method } \\
\cline { 4 - 6 } & & & & $\begin{array}{l}\text { Repair of Original } \\
\text { Lesion Site }\end{array}$ & Nerve Transfer in Wrist \\
\hline I & $\begin{array}{l}10 \mathrm{M} / \mathrm{I} \mathrm{F} \\
8 \mathrm{M} / 3 \mathrm{~F}\end{array}$ & $\begin{array}{l}34(1 \mathrm{I}-48) \\
27.1(5-44)\end{array}$ & $\begin{array}{l}\text { Arm: 5; Elbow: 4; Proximal forearm: } 2 \\
\text { Arm: 4; Elbow: 5; Proximal forearm: } 2\end{array}$ & $\begin{array}{l}\text { Yes } \\
\text { Yes }\end{array}$ & $\begin{array}{l}\text { Yes } \\
\text { No }\end{array}$ \\
\hline
\end{tabular}

Abbreviations: $M$, male; F, female.

\section{Complete High Ulnar Nerve Injury Cases Group I}

In 11 patients, the ulnar nerve was divided at the upper arm (5 patients), elbow (4 patients), and proximal upper forearm ( 2 patients). Direct suture and repair of the ulnar nerve were performed in 8 patients. The remaining 3 patients had a nerve defect; 2 of these patients underwent sural nerve grafting, and 1 patient underwent allogeneic nerve grafting. Transfer of the pronator quadratus muscle nerve branch to the deep branch of the ulnar nerve was performed with endto-side anastomosis in 2 patients and end-to-end anastomosis in 9 patients.

Typical case 1: a patient presented with a right upper arm laceration and complete division of the ulnar nerve in the distal upper arm (Figure 2). An open wound was observed in the distal upper arm. Emergency debridement and exploration were performed, and complete division of the ulnar nerve in the distal upper arm was confirmed. Four days later, no signs of infection were observed in the wound, and then direct fascicular suture was performed in the injured part of the ulnar nerve. The wound was closed. Direct fascicular suture was performed with transfer of the nerve branch of the pronator quadratus muscle using endto-side anastomosis (Figure 2C-F).

Typical case 2: a patient presented with an open wound caused by machinery trauma and complete division of the ulnar nerve in the left distal upper arm (Figure 3). Emergency debridement, hemostasis, and closure of the wound were performed. Eight days later, no signs of infection were observed in the wound, and direct fascicular suture was performed at the injured site of the ulnar nerve. The wound was closed. Then, a longitudinal incision was made on the volar side of the left wrist to expose the deep and superficial branches of the ulnar nerve and the anterior interosseous nerve branch of the pronator quadratus muscle. The anterior interosseous nerve branch of the pronator quadratus muscle was divided at the farthest distal end, and the deep branch of the ulnar nerve was separated non-invasively and then divided at the proximal end. Transfer of the pronator quadratus muscle nerve branch to the ulnar nerve deep branch was performed using end-to-end anastomosis (Figure $3 \mathrm{C}-\mathrm{H}$ ).

Typical case 3: a patient presented with a knife cut and complete division of the ulnar nerve in the proximal left forearm (Figure 4A). Emergency debridement, hemostasis, and closure of the wound were performed (Figure 4B). Eight days later, a longitudinal incision was made on the volar side of the left wrist to expose the deep and superficial branches of the ulnar nerve and the anterior interosseous nerve branch of the pronator quadratus muscle. The anterior interosseous nerve branch of the pronator quadratus muscle was divided at the farthest distal end, and the deep branch of the ulnar nerve was separated noninvasively and then divided at the proximal end. Transfer of pronator quadratus muscle nerve branch to the ulnar nerve deep branch was performed using end-to-end anastomosis (Figure 4C-F).

Typical case 4: a patient presented with a machinery laceration and an open wound in his left proximal forearm. Complete division of the ulnar nerve in the upper third of the forearm with an approximate $3-\mathrm{cm}$ defect was noted (Figure 5). Emergency debridement, hemostasis, and closure of the wound were performed. Seven days later, no signs of infection were observed in the wound, and autologous sural nerve grafting was performed for bridging repair of the ulnar nerve defect (Figure 5C). The wound was closed. Then, the deep and superficial branches of the ulnar nerve and the anterior interosseous nerve branch of the pronator quadratus muscle were exposed on the volar side of the right wrist. The anterior interosseous nerve branch of the pronator quadratus muscle was divided at the farthest distal end, and the deep branch of the ulnar nerve was separated non-invasively and then divided at the proximal end. Transfer of the pronator quadratus muscle nerve branch to the ulnar nerve deep branch was performed using end-to-end anastomosis (Figure 5D-F). 


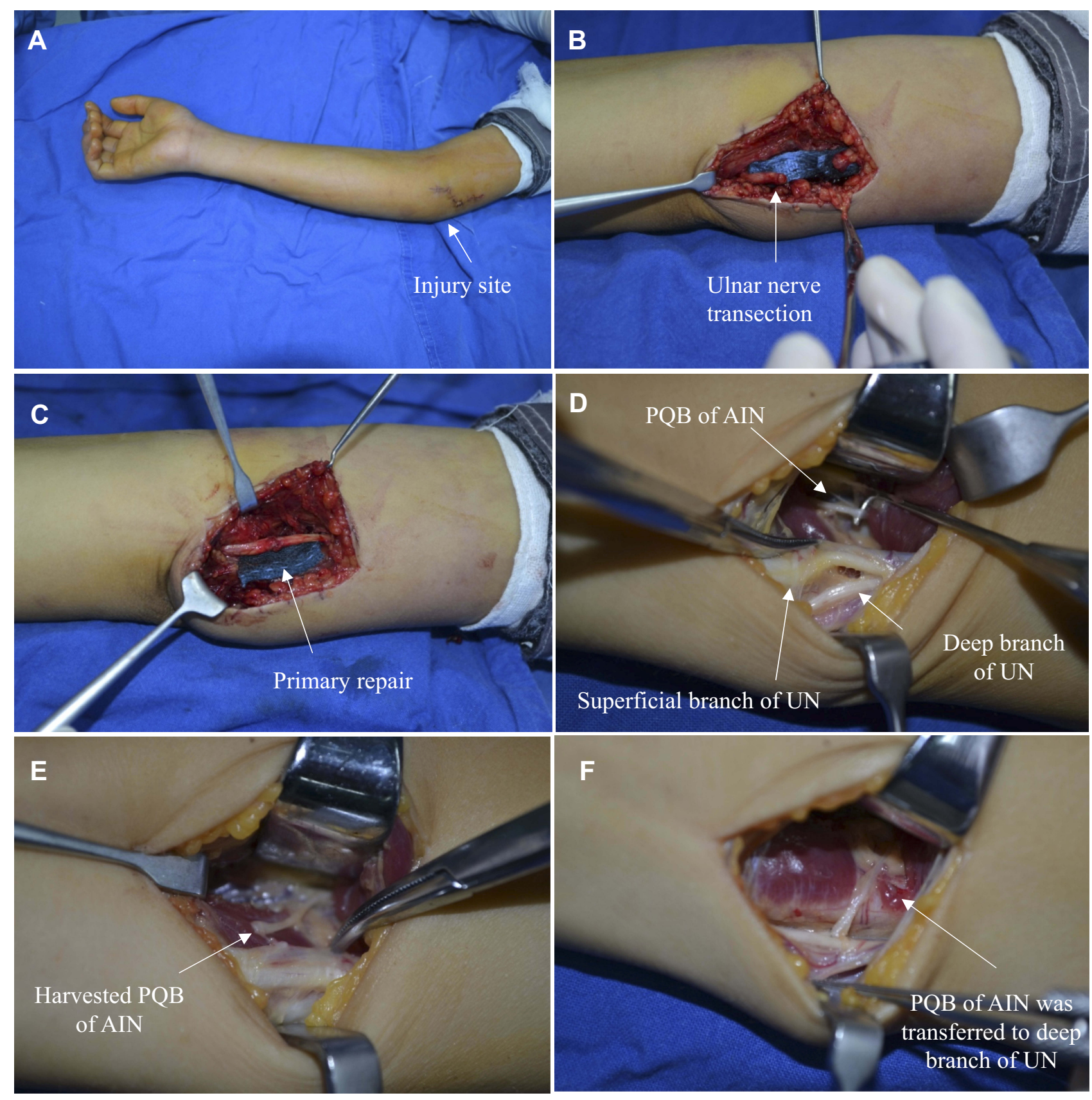

Figure 2 Typical case I in group I an I I-year-old male presented with a laceration in the right upper arm. (A) An open wound was observed in the distal upper arm; (B) Complete division of the ulnar nerve in the distal upper arm; (C) Direct fascicular suture of the ulnar nerve injury site; (D) The deep and superficial branches of the ulnar nerve and the anterior interosseous nerve branch of the pronator quadratus muscle were exposed in the wrist incision; (E) The anterior interosseous nerve branch of the pronator quadratus muscle was divided at the farthest distal end; (F) Transfer of the pronator quadratus muscle nerve branch to the ulnar nerve deep branch was performed using end-to-side anastomosis.

Abbreviations: UN, ulnar nerve; AIN, anterior interosseous nerve; PQB, pronator quadratus branch.

\section{Group II}

In 11 patients, the ulnar nerve was divided at the upper arm (4 patients), elbow (5 patients), and proximal upper forearm (2 patients). Direct suture and repair of the ulnar nerve were performed in 9 patients, and 2 patients with nerve defects underwent sural nerve grafting.
Typical case 5: a patient presented with a glass-induced laceration in the right proximal forearm from 33 days ago. Preoperative examination and electromyography showed complete division of the ulnar nerve. The patient requested to only repair the primary injured part of the ulnar nerve. Intraoperative exploration revealed that the ulnar nerve 

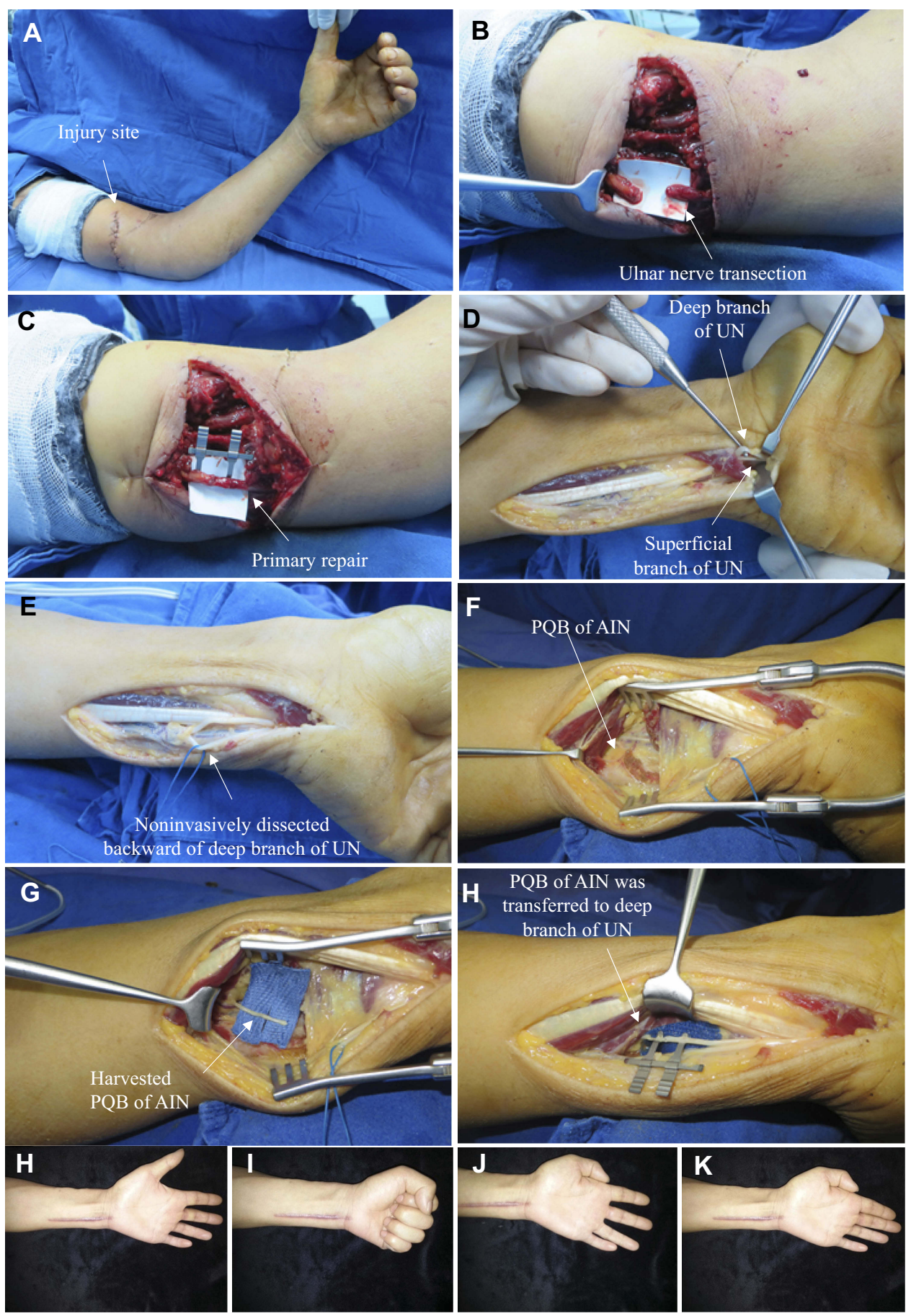

Figure 3 Typical case 2 in group I Direct fascicular suture was performed together with transfer of the nerve branch of the pronator quadratus muscle to the deep branch of the ulnar nerve. (A) An open wound was observed in the distal upper arm; (B) Complete division of the ulnar nerve in the distal upper arm; (C) Direct fascicular suture and repair were performed at the injured site of the ulnar nerve; (D) The deep and superficial branches of the ulnar nerve and the anterior interosseous nerve branch of the pronator quadratus muscle were exposed in the wrist incision; (E) The deep branch of the ulnar nerve was separated non-invasively; (F) The anterior interosseous nerve branch of the pronator quadratus muscle was exposed in the wrist incision; (G) The anterior interosseous nerve branch of the pronator quadratus muscle was divided at the farthest end; (H) Transfer of the pronator quadratus muscle nerve branch to the ulnar nerve deep branch was performed using end-to-end anastomosis; (I-L) Follow-up results after surgery: (I, J) Finger flexion, and extension were normal; $(\mathbf{K}, \mathbf{L})$ The function of the intrinsic muscles of the hand was tested by abduction and adduction motions.

Abbreviations: UN, ulnar nerve; AIN, anterior interosseous nerve; PQB, pronator quadratus branch.

was completely divided in the proximal forearm, and the end of the ulnar nerve was retracted (Figure 6A). No obvious nerve defect was detected by stretching the nerve (Figure 6B). Only fascicular suture of the ulnar nerve at the injury site and closure of the wound were performed (Figure 6C and D). The nerve branch of the 

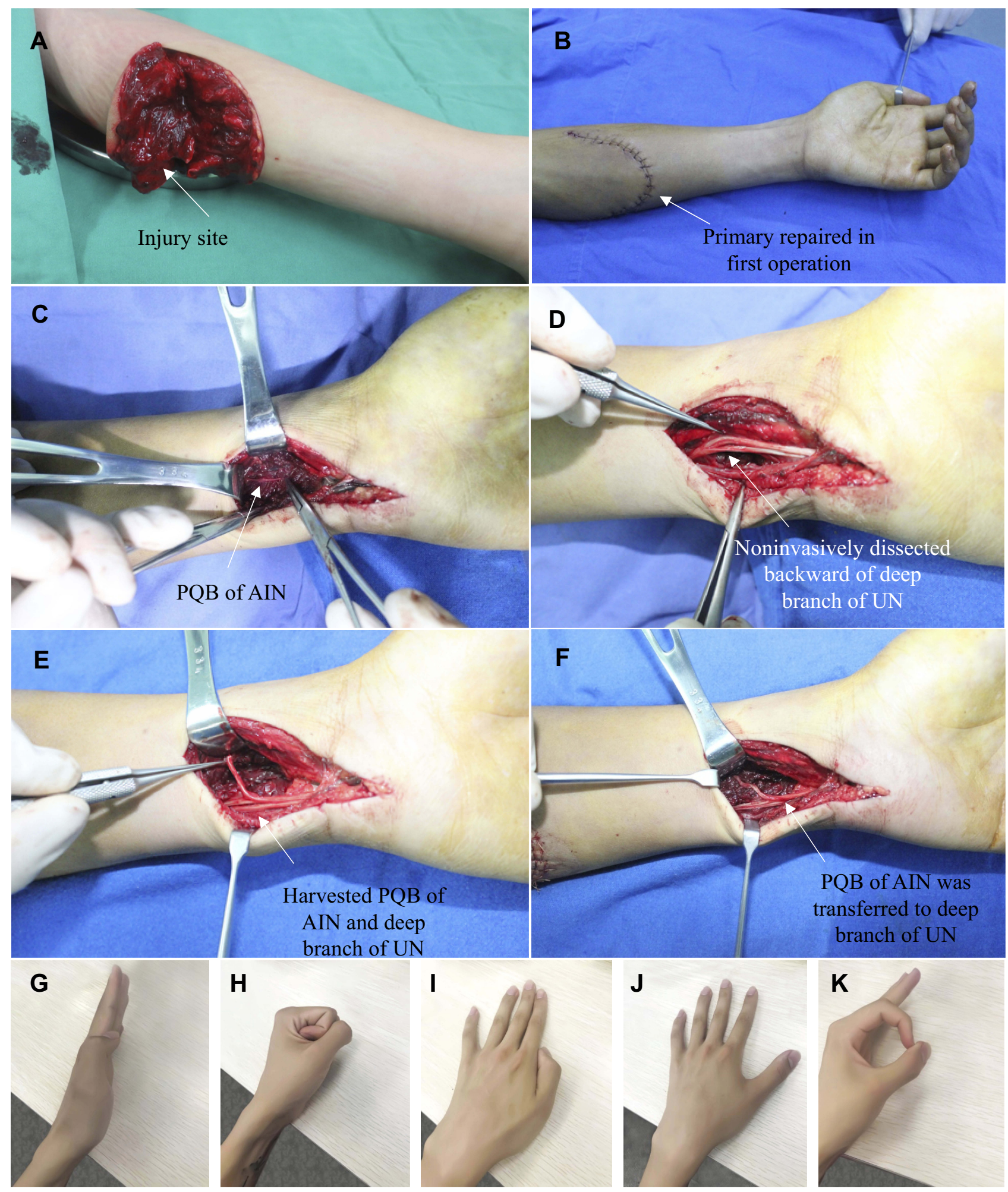

Figure 4 Typical case 3 in group I a 19-year-old male presented with a knife cut in the left proximal forearm. Direct fascicular suture was performed in the injured site of the ulnar nerve. Transfer of the pronator quadratus muscle nerve branch to the ulnar nerve deep branch was performed in the wrist. (A) An open wound and complete division of the ulnar nerve were observed in the proximal forearm; (B) Emergency debridement, hemostasis, and closure of the wound were performed; (C) The anterior interosseous nerve branch of the pronator quadratus muscle was exposed in the wrist incision; (D) The deep and superficial branches of the ulnar nerve were exposed in the wrist incision; (E) The anterior interosseous nerve branch of the pronator quadratus muscle was divided at the farthest end, and the deep branch of the ulnar nerve was separated non-invasively and then divided at the proximal end; (F) Transfer of the pronator quadratus muscle nerve branch to the ulnar nerve deep branch was performed using end-to-end anastomosis; (G-K) Follow-up after surgery: $(\mathbf{G}, \mathbf{H})$ Finger flexion and extension movements were normal; (I, J) The function of the intrinsic muscles of the hand was tested by abduction and adduction motions; $(\mathbf{K})$ Negative Froment's sign.

Abbreviations: UN, ulnar nerve; AIN, anterior interosseous nerve; $\mathrm{PQB}$, pronator quadratus branch. 

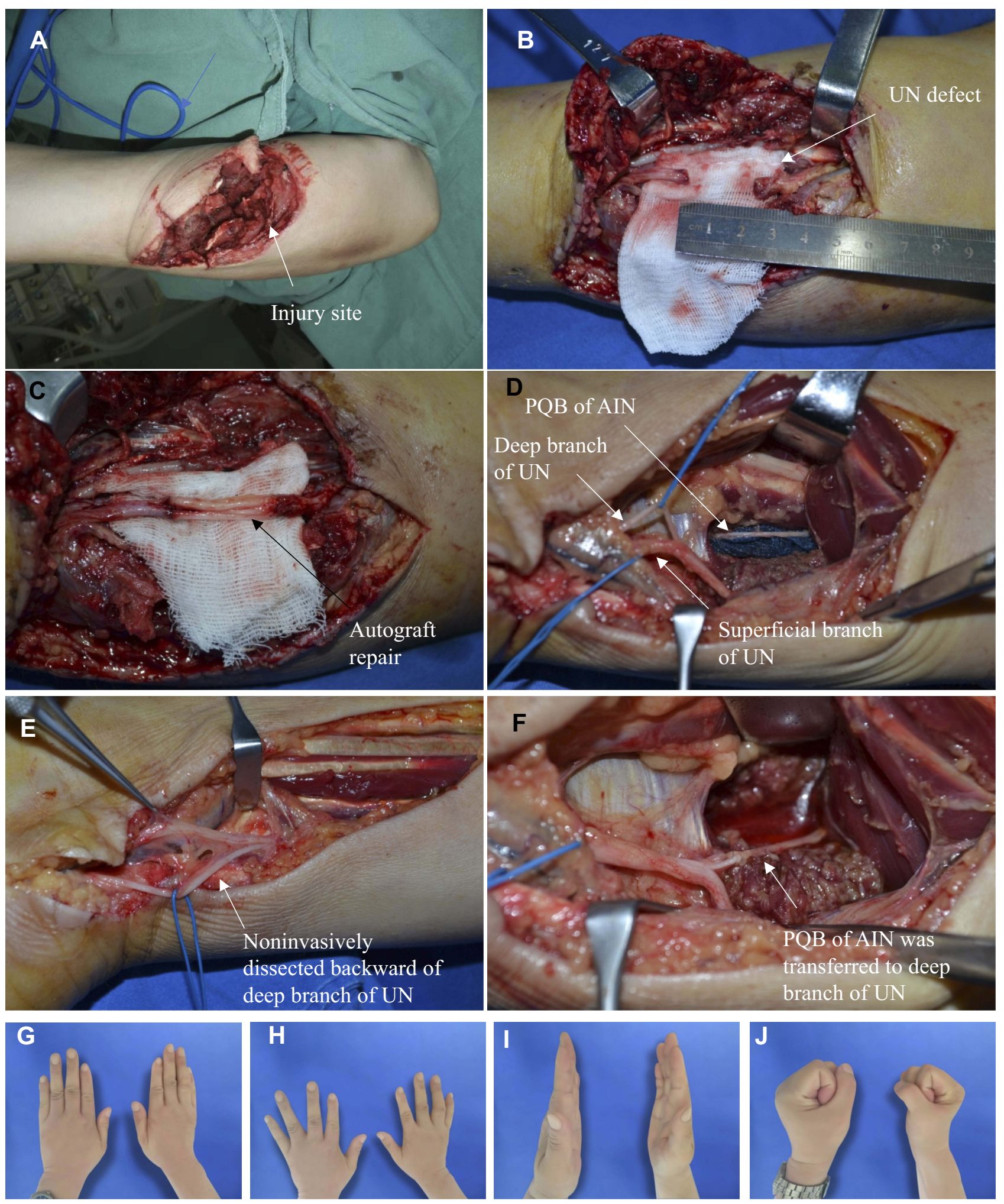

Figure 5 Typical case 4 in group I a 40-year-old male presented with a machinery laceration in the right proximal forearm. (A) An open wound was observed in the distal forearm. Emergency debridement, hemostasis, and closure of the wound were performed; (B) Complete division of the ulnar nerve with an approximate 3-cm defect was noted in the upper third of the forearm; (C) Autologous sural nerve grafting was performed for bridging repair of the ulnar nerve defect; (D) The deep and superficial branches of the ulnar nerve and the anterior interosseous nerve branch of the pronator quadratus muscle were exposed in the wrist incision; (E) The ulnar nerve deep branch was separated retrogradely and non-invasively to the proximal end; $\mathbf{( F )}$ Transfer of the pronator quadratus muscle nerve branch to the ulnar nerve deep branch was performed using end-to-end anastomosis; $(\mathbf{G}-\mathbf{J})$ Follow-up after surgery: $(\mathbf{G}, \mathbf{H})$ The function of the intrinsic muscles of the hand was determined to be normal by abduction and adduction motions of fingers $\mathbf{I}-5$; (I, J) Finger flexion and extension movements were normal.

Abbreviations: UN, ulnar nerve; AIN, anterior interosseous nerve; $\mathrm{PQB}$, pronator quadratus branch. 
pronator quadratus muscle was not transposed to the deep branch of the ulnar nerve at the wrist level.

\section{Follow-Up and Evaluation}

The patients received the scheduled follow-up. The muscle strength of the first dorsal interosseous muscles of the affected side (examining abduction motion of the index finger) was measured $6,12,24,36$, and 48 months after surgery using the Medical Research Council (MRC) muscle strength rating system modified by Brandsma ${ }^{14}$ (Table 2). The MRC scale for sensory recovery modified by Mackinnon and Dellon was used to assess the sensation function of the pulp of the little finger ${ }^{15}$ (Table 3 ). The grip and pinch (tripod pinch) strengths of both hands were measured. The claw hand deformity, Froment's sign, Wartenberg's sign, atrophy of the intrinsic muscles (mainly including the first, third, and fourth dorsal interosseous muscles), and the degree of rotation of the affected forearm were evaluated. SPSS software (Version 21, American IMB company) was used for the statistical analysis.

According to the Highet-Zachary scheme ${ }^{16}$, the mean scores for sensation and muscle strength were calculated (M0-M5 correspond to scores of 0-5, respectively; S0, S1, $\mathrm{S} 2, \mathrm{~S} 3, \mathrm{~S} 3+, \mathrm{S} 4$ correspond to scores of 0-5, respectively). The function of the affected upper limb was scored using
Table 2 MRC Muscle Strength Grading Modified by Brandsma

\begin{tabular}{|c|c|c|c|}
\hline Grade & & Group I & Group II \\
\hline M0: & No contraction & & \\
\hline MI: & Muscle tremor or contraction & & \\
\hline M2: & $\begin{array}{l}\text { Full range active movement } \\
\text { without gravity resistance }\end{array}$ & $n=1$ & $n=3$ \\
\hline M3: & $\begin{array}{l}\text { Able to carry out active } \\
\text { movement against gravity, not to } \\
\text { resist resistance }\end{array}$ & & $n=6$ \\
\hline M4: & $\begin{array}{l}\text { Able to carry out active } \\
\text { movement against gravity and } \\
\text { resist light resistance }\end{array}$ & $n=7$ & $n=1$ \\
\hline M5: & Normal & $n=3$ & $n=1$ \\
\hline
\end{tabular}

the Disabilities of the Arm, Shoulder, and Hand (DASH) scale. ${ }^{17}$ A paired sample $t$-test was used to compare the data (the time from injury to surgery, follow-up period, sensory score on the Highet Scale, muscle strength score on the Highet Scale, handgrip strength, pinch strength, and DASH score) between group I and group II.

Only the numbers of patients with Froment's sign, Wartenberg's sign, atrophy of the first, third, and fourth dorsal interosseous muscles, and claw hand deformity were compared because the sample size in each group was small $(n<40)$.
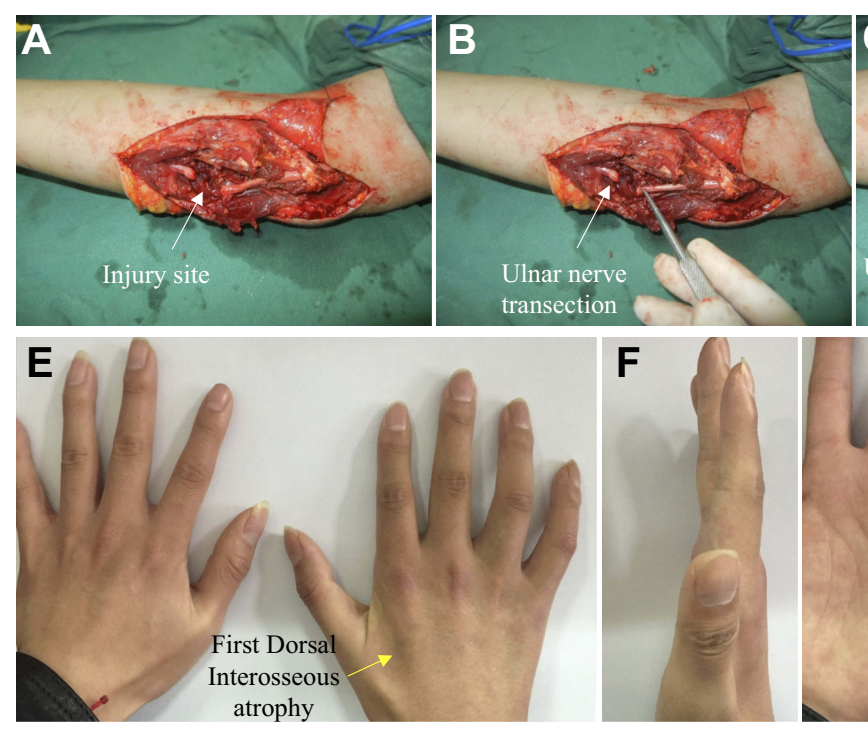
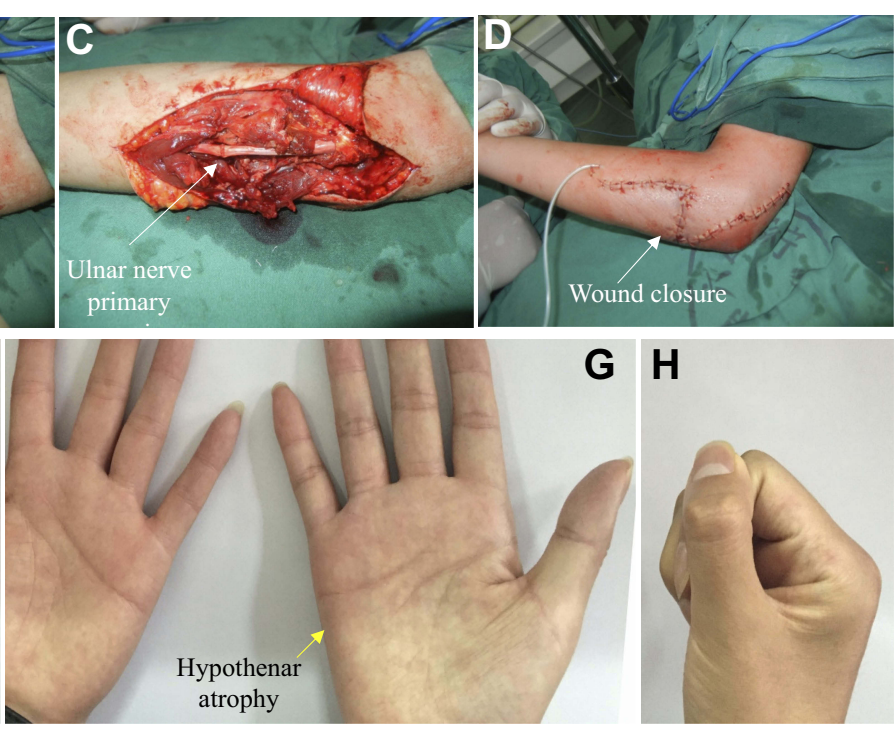

G H

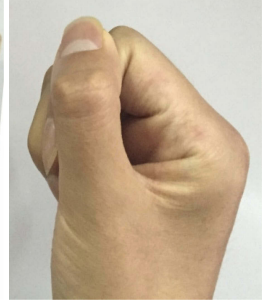

Figure 6 Typical case 5 in group II: Ulnar nerve division was confirmed in the proximal forearm. Only fascicular suture of the injury site of the ulnar nerve was performed; the nerve branch of the pronator quadratus muscle was not transposed to the deep branch of the ulnar nerve at the wrist level. (A) A wound in the proximal forearm; (B) The ulnar nerve was completely divided in the proximal forearm, and the end of the ulnar nerve was retracted. No obvious nerve defect was detected by stretching the nerve; (C) Fascicular suture to repair the ulnar nerve; (D) Primary suture of the wound; (E-H) Follow-up after surgery: (E, G) Obvious atrophy of the first interosseous dorsal muscle and the hypothenar muscles; $(\mathbf{F}, \mathbf{H})$ Flexion and extension of fingers were normal.

Abbreviations: UN, ulnar nerve; AIN, anterior interosseous nerve; $\mathrm{PQB}$, pronator quadratus branch. 
Table 3 Sensory Nerve Recovery Grading Modified by Mackinnon and Dellon

\begin{tabular}{|c|c|c|c|}
\hline \multicolumn{2}{|c|}{ Grade } & \multirow[t]{2}{*}{ Group I } & \multirow[t]{2}{*}{ Group II } \\
\hline So: & $\begin{array}{l}\text { Absence of sensibility in the } \\
\text { autonomous zone of the nerve }\end{array}$ & & \\
\hline SI: & $\begin{array}{l}\text { Recovery of deep cutaneous pain } \\
\text { sensibility within the autonomous } \\
\text { zone of the nerve }\end{array}$ & & \\
\hline S2: & $\begin{array}{l}\text { Partial recovery of superficial pain and } \\
\text { tactile sensibility within the } \\
\text { autonomous area of the nerve }\end{array}$ & $n=1$ & $n=3$ \\
\hline S3: & $\begin{array}{l}\text { Recovery of superficial cutaneous pain } \\
\text { and tactile sensibility throughout the } \\
\text { autonomous area without hyperalgesia. } \\
\text { S2PD }>15 \mathrm{~mm}, \mathrm{M} 2 \mathrm{PD}>7 \mathrm{~mm}\end{array}$ & $n=3$ & $n=3$ \\
\hline S3+: & $\begin{array}{l}\text { Recover to S3 level, and some } \\
\text { recovery of two point's discrimination. } \\
\text { S2PD: 7-15 mm, M2PD: 4-7 mm }\end{array}$ & $n=3$ & $n=4$ \\
\hline S4: & $\begin{array}{l}\text { Complete recovery. S2PD: } 2-6 \mathrm{~mm} \text {, } \\
\text { M2PD: } 2-3 \mathrm{~mm}\end{array}$ & $n=4$ & $n=1$ \\
\hline
\end{tabular}

Abbreviations: M2PD, moving two-point discrimination; S2PD, static two-point discrimination.

\section{Results}

\section{Surgery and Functional Recovery in the} Patients

All 22 patients underwent successful surgical procedures according to the preoperative design. At the 48-month follow-up for typical case 1, the muscle strength of the flexor muscle of the wrist was assigned a score of 5 , and flexion and extension of the affected finger were completely normal (Figure 7A-C). The adduction motion of the ring finger was mildly restricted, and the adduction motion of the small finger was limited (positive Wartenberg's sign), but abduction motion was normal, the muscle strength of the first dorsal interosseous muscle was scored as M5 (Figure 7D), and atrophy of the right hypothenar muscles was not evident (Figure 7E). Froment's sign was negative and atrophy of the first interosseous dorsal muscle was not evident (Figure 7F). Normal forearm pronation and supination suggested that the pronator teres muscle fully compensated for the rotation function of the pronator quadratus muscle (Figure $7 \mathrm{G}-\mathrm{H}$ ). The grip strength of the affected hand (the patient was right-hand dominant) was
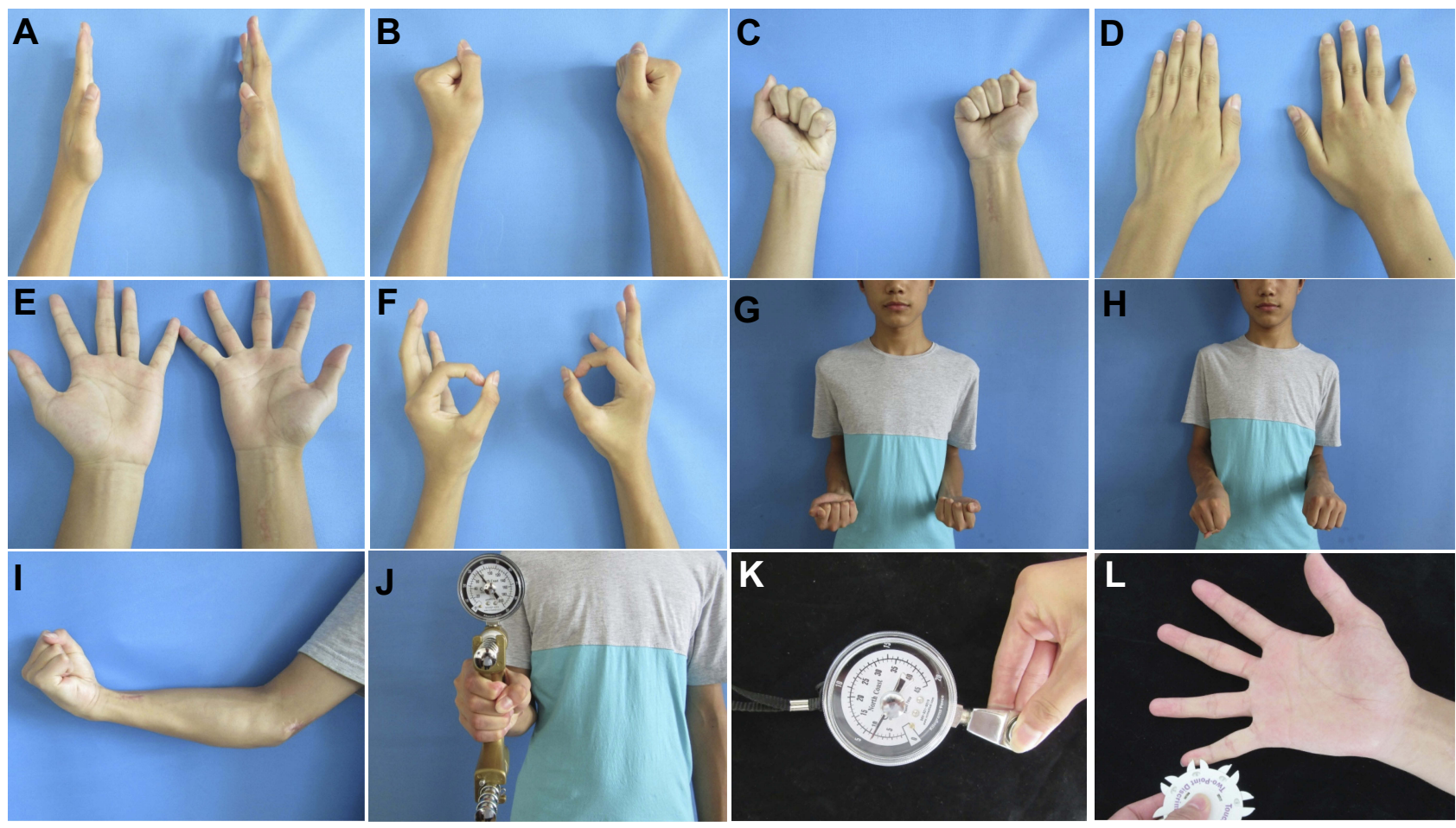

Figure 7 The results of typical case I at the 48-month follow-up. (A-C) Completely normal finger flexion and extension; (D-F) The function of the intrinsic muscles of the hand was determined to be normal by observing abduction motions of fingers I-5; (F) Froment's sign was negative, and atrophy of the first interosseous dorsal muscle was not apparent; (G, H) Normal forearm pronation and supination; (I) Flexor muscle strength was scored as level 5; (J) The grip strength of the affected hand (the patient was right-hand dominant) was $35 \mathrm{~kg}$; (K) The pinch strength of the affected hand was $9.5 \mathrm{~kg}$; (L) On the affected side, static two-point discrimination of the fingertips was equal to $6 \mathrm{~mm}$. 
$35 \mathrm{~kg}$ and the pinch strength was $9.5 \mathrm{~kg}$ (Figure $7 \mathrm{~J}-\mathrm{K}$ ). Sensory recovery was achieved at the S4 level (Figure 7L).

At the 24-month follow-up of typical case 2, flexion and extension of the left fingers were normal, and atrophy of the left hypothenar muscles was not evident (Figure 3H and I). The abduction motion of the little finger was normal, but the adduction motion was limited (Figure 3J-K). Froment's sign was negative and atrophy of the first interosseous dorsal muscle was not evident. The grip strength of the affected hand (the patient was right-hand dominant) was $28 \mathrm{~kg}$. The pinch strength of the affected hand was $8 \mathrm{~kg}$. On the affected side, static two-point discrimination of the fingertips was greater than $15 \mathrm{~mm}$. Sensory recovery was achieved at the S3 level.

At the 46-month follow-up of typical case 3, flexion and extension of the fingers on the left hand were normal (Figure 4G-H). The abduction motion of the little finger was normal, but the adduction motion was limited (Figure 4I and J). Atrophy of the first dorsal interosseous muscle was not evident, and Froment's sign was negative (Figure 4K). The grip strength of the affected hand (the patient was right-hand dominant) was $42 \mathrm{~kg}$. The pinch strength of the affected hand was $10.5 \mathrm{~kg}$. On the affected side, static two-point discrimination of the fingertips was equal to $5 \mathrm{~mm}$. Sensory recovery was achieved at the S4 level.

At the 47-month follow-up of typical case 4, the intrinsic muscles of the right hand were determined to be normal by observing adduction and abduction motions of fingers 1-5. Wartenberg's sign was negative (Figure 5G and H). No obvious atrophy was noted in the first, third, and fourth dorsal interosseous muscles (Figure $5 \mathrm{G}$ and $\mathrm{H}$ ). Finger flexion and extension were normal (Figure 5I and J). Froment's sign was negative. The grip strength of the affected hand (the patient was right-hand dominant) was $48 \mathrm{~kg}$. The pinch strength of the affected hand was $10.5 \mathrm{~kg}$. On the affected side, static two-point discrimination of the little fingertips was equal to $8 \mathrm{~mm}$. Sensory recovery was achieved at the $\mathrm{S} 3+$ level.

At the 47-month follow-up of typical case 5 in group II, obvious atrophy was observed in the first dorsal interosseous muscle and the hypothenar muscles (Figure 6E and G). Finger flexion and extension were normal (Figure $6 \mathrm{~F}$ and $\mathrm{H}$ ). Wartenberg's sign and Froment's sign were positive. The grip strength of the affected hand (the patient was right-hand dominant) was $25 \mathrm{~kg}$. The pinch strength of the affected hand was $6 \mathrm{~kg}$. On the affected side, static two-point discrimination of the little fingertips was greater than $15 \mathrm{~mm}$. Sensory recovery was achieved at the S3 level.

\section{Follow-Up and Evaluation}

The first follow-up visit was scheduled 6 months after surgery and the final follow-up was in May 2018.

Table 2 shows the follow-up results for the muscle strength of the first interosseous muscles. In group I, the muscle strength in 10 of 11 patients was recovered to the M4 or M5 level. In group II, the muscle strength in 2 of 11 patients was recovered to the M4 or M5 level.

The follow-up results for skin sensation of the little finger pulp are shown in Table 3. Sensory recovery was achieved at the S2 or higher in both groups. Sensory recovery reached the $\mathrm{S} 3+$ level or higher in $7(7 / 11)$ patients in group I and in $5(5 / 11)$ patients in group II.

The function evaluation of the patients is shown in Table 4. No significant differences were identified between groups I and II in the time interval from injury to surgery and the follow-up period $(\mathrm{p}>0.05)$. No restrictions were noted for rotation of the affected forearm in both groups. No significant difference was found in the mean score for sensory function according to the Highet Scale between the two groups. The muscle strength of the first interosseous muscle was significantly better in group I than that in group II $(p=0.010)$. The number of patients with atrophy of the first, third, and fourth dorsal muscles or claw hand deformity was lower in group I than that in group II. The grip strength, pinch strength, and DASH score were significantly better in group I than those in group II $(\mathrm{p}<0.01)$.

In group I, transfer of the pronator quadratus muscle nerve branch to the deep branch of the ulnar nerve was performed with end-to-side anastomosis in 2 patients and end-to-end anastomosis in 9 patients. Because of the small sample size of the data, there was no statistical comparison between the two kinds of nerve anastomosis. There were 2 cases of sural nerve transplantation in two groups each because of the nerve defect. Due to the small size of data, there was no statistical comparison between direct suture of ulnar nerve and sural nerve transplantation. The outcome of these minority patients was added in Table 5.

\section{Discussion}

Atrophy of the intrinsic muscles of the hand is difficult to avoid in patients undergoing routine repair for high ulnar nerve injury. In severe cases, the claw hand deformity is a consequence that affects hand function and appearance. ${ }^{18,19}$ The challenge in repairing complete division of the ulnar nerve 
Table 4 Outcome of Patients (Based on the Latest Follow-Up)

\begin{tabular}{|c|c|c|c|}
\hline & Group I & Group II & p value \\
\hline Time interval between injury and operation (day), mean (range) & $10.18(4-23)$ & $3.73(0-33)$ & 0.128 \\
\hline Follow up time (month), mean (range) & $39.73(22-60)$ & $33.27(17-57)$ & 0.142 \\
\hline Highet Scale for sensory, mean (standard deviation) & $3.91(1.04)$ & $3.27(1.00)$ & 0.132 \\
\hline Highet Scale for motor, mean (standard deviation) & $4.09(0.83)$ & $3.00(0.89)$ & 0.010 \\
\hline Froment sign, positive & $2 / 11$ & $5 / 11$ & - \\
\hline Wartenberg sign, positive & $7 / 11$ & $8 / 11$ & - \\
\hline Flexor carpi ulnaris atrophy, positive & $2 / 11$ & $\mathrm{I} / \mathrm{II}$ & - \\
\hline First interosseous dorsal muscular atrophy, positive & $3 / 11$ & $7 / 11$ & - \\
\hline Third, fourth interosseous muscle atrophy, positive & $6 / 11$ & $8 / 11$ & - \\
\hline Clawing deformity, positive & $3 / 11$ & $6 / 11$ & - \\
\hline Grip (\%), mean (range) & $75(50-85)$ & $63(45-75)$ & 0.007 \\
\hline Pinch (\%), mean (range) & $66(50-75)$ & $56(45-65)$ & 0.003 \\
\hline DASH, mean (range) & $8.82(0-25)$ & $22.09(4-45)$ & 0.009 \\
\hline
\end{tabular}

Abbreviation: DASH, disabilities of the arm, shoulder, and hand.

Table 5 Outcome of Minority Patients of End-to-Side Anastomosis and Sural Nerve Grafting (Based on the Latest Follow-Up)

\begin{tabular}{|c|c|c|c|}
\hline & \multicolumn{2}{|l|}{ Group I } & \multirow{2}{*}{$\begin{array}{l}\text { Group II } \\
\text { Sural } \\
\text { Nerve } \\
\text { Grafting } \\
(n=2)\end{array}$} \\
\hline & $\begin{array}{l}\text { End-to-Side } \\
\text { Anastomosis } \\
(n=2)\end{array}$ & $\begin{array}{l}\text { Sural } \\
\text { Nerve } \\
\text { Grafting } \\
(n=2)\end{array}$ & \\
\hline Sensory, range & $S 3+\sim 54$ & S2 S3 & $\mathrm{S} 2 \sim \mathrm{S} 3+$ \\
\hline Muscle strength, range & M4 & M2 M4 & $M 2 \sim M 3$ \\
\hline Froment sign, positive & $1 / 2$ & $1 / 2$ & $1 / 2$ \\
\hline Wartenberg sign, positive & $2 / 2$ & $1 / 2$ & $2 / 2$ \\
\hline $\begin{array}{l}\text { Flexor carpi ulnaris atrophy, } \\
\text { positive }\end{array}$ & $0 / 2$ & $1 / 2$ & $1 / 2$ \\
\hline $\begin{array}{l}\text { First interosseous dorsal } \\
\text { muscular atrophy, positive }\end{array}$ & $1 / 2$ & $1 / 2$ & $2 / 2$ \\
\hline $\begin{array}{l}\text { Third, fourth interosseous } \\
\text { muscle atrophy, positive }\end{array}$ & $1 / 2$ & $2 / 2$ & $2 / 2$ \\
\hline Clawing deformity, positive & $1 / 2$ & $1 / 2$ & $2 / 2$ \\
\hline Grip (\%), range & $70 \sim 75$ & $50 \sim 70$ & $50 \sim 65$ \\
\hline Pinch (\%), range & $70 \sim 75$ & $50 \sim 65$ & $45 \sim 60$ \\
\hline DASH, range & $9 \sim 10$ & $12 \sim 25$ & $25 \sim 45$ \\
\hline
\end{tabular}

Abbreviation: DASH, disabilities of the arm, shoulder, and hand.

is to identify and implement effective measures to shorten the nerve regeneration path and to restore innervation of the intrinsic muscles of the hand before severe muscle atrophy occurs. $^{20}$ In this work, based on nerve magnified regeneration, the motor nerve transfer technique was used to shorten the nerve regeneration path and the time to reinnervation of the intrinsic muscles, substantially reducing atrophy of these muscles and providing better hand function.

In this work, long-term follow-up data of a repair method for complete high ulnar nerve injury confirmed the phenomena of nerve-magnified regeneration. The distal part of the anterior interosseous nerve is a mixed nerve with sensory fibres. ${ }^{13}$ Although previous studies have shown that the axon size of this branch of the anterior interosseous nerve of the pronator quadratus muscle is quite different from that of the ulnar nerves, ${ }^{12}$ we found that transfer of the pronator quadratus muscle nerve branch can lead to innervation of multiple target muscles that are usually innervated by the deep branch of the ulnar nerve, such as the first interosseous muscle and the hypothenar muscle described in typical cases $1-4$. The data described above confirmed that nerve-magnified regeneration can occur and are reliable.

The repair method for high ulnar nerve injury in this work can effectively restore hand function. The mean scores (the Highet Scale) for muscle strength of the first dorsal interosseous muscle were significantly different between group I and group II, indicating that the modified repair method can effectively improve the intrinsic muscle strength of the hand compared with the traditional method. The protective skin sensation of the little finger and the ulnar side of the palm has important value for preventing burns of the affected hand and improving the coordination ability of the hand, ${ }^{21}$ thus enhancing a patient's confidence.

Here, transfer of the pronator quadratus muscle nerve branch effectively reduced atrophy of the intrinsic muscles of the hand, the rate of positive Froment's sign and incidence of the claw hand deformity (Table 1), thus improving the hand function of the patients. In typical cases 1,3 , and 4, the first dorsal interosseous muscle was in good condition without obvious atrophy. The time to reinnervation of the muscles of the hand was also reduced. Group I included fewer patients with atrophy of the first interosseous muscle and 
a positive Froment's sign compared to group II. First, as anatomical studies suggest, the lower number of axons of the nerve branch of the pronator quadratus muscle ${ }^{12}$ may cause incomplete innervation. Schenck et $\mathrm{al}^{12}$ showed that the ratio of the axon size of the anterior interosseous nerve to that of the deep branch of the ulnar nerve is 1:4.8, while Jiang et $\mathrm{al}^{8}$ demonstrated that the capacity for nerve amplification is 1:3-4. Therefore, incomplete innervation is possible. Second, some of the intrinsic muscles of the hand experienced irreversible atrophy before complete reinnervation by the pronator quadratus muscle nerve branch. ${ }^{22}$ Finally, the third and fourth dorsal interosseous muscles may be more prone to atrophy or atrophy than the first dorsal interosseous muscles. Many patients in group I (7/11) and group II (8/11) presented a positive Wartenberg's sign, the little finger thus always tends to stay in the abduction position. ${ }^{23,24}$ Long-term abduction causes ligament loosening and avulsion of the coronal bundles on the radial side of the extensor tendon hood of the little finger, further aggravating the deformity. ${ }^{25}$ This deformity can be corrected by tendon transfer. ${ }^{23,24}$

In the current work, grip strength, pinch strength, and the DASH score were significantly better in group I than those in group II (Table 1). Both intrinsic and extrinsic muscles are involved in grip strength and pinch strength. ${ }^{26,27}$ Among these muscles, pinch strength (tripod pinch) requires strong contraction of the adductor pollicis muscle and the first dorsal interosseous muscle, which are innervated by the deep branch of the ulnar nerve. ${ }^{28}$ Therefore, good pinch strength indicates good reinnervation of the ulnar nerve to these muscles. Grip strength and pinch strength reflect practical functions in patients' daily lives and are negatively correlated with DASH score (a higher DASH score corresponds to worse function). ${ }^{29,30}$ In this work, good recovery of grip strength and pinch strength allowed patients to resume their daily life and work activities, resulting in high patient satisfaction with treatment.

Further studies are needed to clarify the indications for end-to-end anastomosis or end-to-side anastomosis with transfer of the nerve branch of the pronator quadratus muscle to the deep branch of the ulnar nerve to repair high ulnar nerve injury. Studies have shown that end-to-side anastomosis may result in innervation by the 2 motor nerves. ${ }^{10}$ The intrinsic muscles can be innervated by the pronator quadratus muscle branch in the early stage and are expected to receive innervation from the regenerated ulnar nerve itself in the later stage. ${ }^{31}$ According to the follow-up data, the degree of sensory recovery was found in the ulnar nerve-innervated area can reach the S2 level or higher, suggesting that some regenerated nerve fibres indeed reach the distal effector after repair of the injured part of the ulnar nerve, even though the regeneration path is long. Therefore, end-to-side anastomosis can achieve an additive effect due to ulnar nerve regeneration.

\section{Conclusion}

For the treatment of complete high ulnar nerve injury, this work proposes a repair method based on nerve magnified regeneration, ie, first repairing the ulnar nerve at the injured site, and then transposing the nerve branch of the pronator quadratus muscle to the deep branch of the ulnar nerve at the wrist level. This method is a feasible technique that not only validates nerve magnified regeneration in clinical practice but also mitigates loss of function in the nerve donor area. Therefore, this technique substantially reduces atrophy of the intrinsic muscles of the hand and provides better hand function. We advocate this technique as a routine strategy in clinical practice.

\section{Acknowledgments}

This work was supported by Natural Science Foundation of China (NSFC), Grant number: 81671928; Special Project for Major Diseases' Prevention and Treatment (Trauma Repair), National Health Commission of China-, Grant number: ZX-01-C2015003; Medical Health Science and Technology Project of Zhejiang Province, Grant number: 2019310846; Australia National Health and Medical Research Council (NHMRC) Grant, Grant number: 1158402; Natural Science Foundation of Ningbo, Grant number: 2019A610279; Public Welfare Project of Ningbo, Grant number: 2019C50044.

\section{Disclosure}

The authors report no conflicts of interest in this work.

\section{References}

1. Gottschalk HP, Bindra RR. Late reconstruction of ulnar nerve palsy. Orthop Clin N Am. 2012;43(4):495-507. doi:10.1016/j.ocl.2012.08.001

2. Nath RK, Mackinnon SE. Nerve transfers in the upper extremity. $J$ Am Soc Surg Hand. 2000;4(3):131-139.

3. Buchthal F, Kühl V. Nerve conduction, tactile sensibility, and the electromyogram after suture or compression of peripheral nerve: a longitudinal study in man. J Neurol Neurosurg Psychiatry. 1979;42 (5):436-451. doi:10.1136/jnnp.42.5.436

4. Post R, de Boer KS, Malessy MJ. Outcome following nerve repair of high isolated clean sharp injuries of the ulnar nerve. PLoS One. 2012;7 (10):e47928. doi:10.1371/journal.pone.0047928

5. Diaz-Garcia RJ, Chung KC. A comprehensive guide on restoring grasp using tendon transfer procedures for ulnar nerve palsy. Hand Clin. 2016;32(3):361-368. doi:10.1016/j.hcl.2016.03.006

6. Battiston B, Lanzetta M. Reconstruction of high ulnar nerve lesions by distal double median to ulnar nerve transfer. J Hand Surg. 1999;24 (6):1185-1191. doi:10.1053/jhsu.1999.1185 
7. Flores LP. Distal anterior interosseous nerve transfer to the deep ulnar nerve and end-to-side suture of the superficial ulnar nerve to the third common palmar digital nerve for treatment of high ulnar nerve injuries: experience in five cases. Arq Neuropsiquiatr. 2011;69(3):519-524. doi:10.1590/S0004-282X2011000400021

8. Bao GJ, Xiao FY, Zhang DY, Zhong GF, Hong BZ. Maximum number of collaterals developed by one axon during peripheral nerve regeneration and the influence of that number on reinnervation effects. Eur Neurol. 2007;58(1):12-20. doi:10.1159/000102161

9. Zhang P, Kou Y, Yin X, Wang Y, Zhang H, Jiang B. The experimental research of nerve fibers compensation amplification innervation of ulnar nerve and musculocutaneous nerve in rhesus monkeys. Artif Cell Blood Sub Immobilization Biotechnol. 2011;39(1):39. doi:10.3109/10731199. 2010.494583

10. Isaacs JE, Cheatham S, Gagnon EB, Razavi A, Mcdowell CL. Reverse end-to-side neurotization in a regenerating nerve. J Reconstr Microsurg. 2008;24(07):489-496. doi:10.1055/s-0028-1088230

11. Wang Y, Zhu S. Transfer of a branch of the anterior interosseus nerve to the motor branch of the median nerve and ulnar nerve. Chin Med J. 1997;110(3):216.

12. Schenck TL, Stewart J, Lin S, Aichler M, Machens HG, Giunta RE. Anatomical and histomorphometric observations on the transfer of the anterior interosseous nerve to the deep branch of the ulnar nerve. J Hand Surg Eur Vol. 2015;34(3):591-596. doi:10.1177/1753193414551909

13. Grafe MW, Kim PD, Rosenwasser MP, Strauch RJ. Wrist denervation and the anterior interosseous nerve: anatomic considerations. J Hand Surg Am. 2005;30(6):1221-1225. doi:10.1016/j.jhsa.2005.06.010

14. Brandsma JW, Schreuders TA, Birke JA, Piefer A, Oostendorp R. Manual muscle strength testing: intraobserver and interobserver reliabilities for the intrinsic muscles of the hand. J Hand Ther. 1995;8 (3):185-190. doi:10.1016/S0894-1130(12)80014-7

15. Mackinnon S, Dellon A. Surgery of the peripheral nerve. New York: Thieme. 1988:119. doi:10.3168/jds.S0022-0302(88)79586-7

16. Basar H, Basar B, Erol B, Tetik C. Comparison of ulnar nerve repair according to injury level and type. Int Orthop. 2014;38(10):2123. doi:10.1007/s00264-014-2430-y

17. Atroshi I, Gummesson C, Andersson B, Dahlgren E, Johansson A. The disabilities of the arm, shoulder and hand (DASH) outcome questionnaire: reliability and validity of the Swedish version evaluated in 176 patients. Acta Orthop Scand. 2000;71(6):613. doi:10. $1080 / 000164700317362262$

18. Gaul JS Jr. Intrinsic motor recovery-A long-term study of ulnar nerve repair. J Hand Surg. 1982;7(5):502. doi:10.1016/S03635023(82)80048-8

19. Vordemvenne T, Langer M, Ochman S, Raschke M, Schult M. Longterm results after primary microsurgical repair of ulnar and median nerve injuries. A comparison of common score systems. Clin Neurol Neurosurg. 2007;109(3):263-271. doi:10.1016/j.clineuro.2006.11.006
20. Tung TH, Mackinnon SE. Nerve transfers: indications, techniques, and outcomes. J Hand Surg Am. 2010;35(2):332-341. doi:10.1016/j. jhsa.2009.12.002

21. Ho ES, Davidge K, Curtis CG, Clarke HM. Sensory outcome in children following microsurgery for brachial plexus birth injury. J Hand Surg Am. 2018;44(2).

22. Carlson BM. The biology of long-term denervated skeletal muscle. Eur J Transl Myol. 2014;24(1):3293. doi:10.4081/bam.2014.1.5

23. Van AJ, Zhu J, Fasel JH, Beaulieu JY. Investigation of radialization and rerouting of the extensor digiti minimi (EDM) in the abduction deformity of the little finger: a cadaver study. Hand. 2011;6 (2):202-205. doi:10.1007/s11552-011-9320-1

24. Voche P, Merle M. Wartenberg's sign. A new method of surgical correction. J Hand Surg Br Eur Vol. 1995;20(1):49-52. doi:10.1016/ S0266-7681(05)80016-6

25. Lourie GM, Lundy DW, Rudolph HP, Bayne LG. Abducted, hyperextended small finger deformity of nonneurologic etiology. $J$ Hand Surg. 1999;24(2):315-319. doi:10.1053/jhsu.1999.0315

26. Kozin SH, Porter S, Clark P, Thoder JJ. The contribution of the intrinsic muscles to grip and pinch strength. J Hand Surg. 1999;24 (1):64-72. doi:10.1053/jhsu.1999.jhsu24a0064

27. Zhang Y, Yin $\mathrm{C}, \mathrm{Hu} \mathrm{L}$, et al. MACF1 overexpression by transfecting the $21 \mathrm{kbp}$ large plasmid PEGFP-C1A-ACF7 promotes osteoblast differentiation and bone formation. Hum Gene Ther. 2018;29 (2):259-270. doi:10.1089/hum.2017.153

28. Barry AJ, Murray WM, Kamper DG. Development of a dynamic index finger and thumb model to study impairment. J Biomech. 2018;77:206-210. doi:10.1016/j.jbiomech.2018.06.017

29. Beumer A, Lindau TR. Grip strength ratio: a grip strength measurement that correlates well with DASH score in different hand/wrist conditions. BMC Musculoskelet Disord. 2014;15(1):1-5. doi:10.1186/1471-2474-15336

30. Klum M, Wolf MB, Hahn P, Leclère FM, Bruckner T, Unglaub F. Predicting grip strength and key pinch using anthropometric data, DASH questionnaire and wrist range of motion. Arch Orthop Trauma Surg. 2012;132(12):1807-1811. doi:10.1007/s00402-0121602-8

31. Baltzer H, Woo A, Oh C, Moran SL. Comparison of ulnar intrinsic function following supercharge end-to-side anterior interosseous-toulnar motor nerve transfer: a matched cohort study of proximal ulnar nerve injury patients. Plast Reconstr Surg. 2016;138(6):1264-1272. doi:10.1097/PRS.0000000000002747
Therapeutics and Clinical Risk Management

\section{Publish your work in this journal}

Therapeutics and Clinical Risk Management is an international, peerreviewed journal of clinical therapeutics and risk management, focusing on concise rapid reporting of clinical studies in all therapeutic areas, outcomes, safety, and programs for the effective, safe, and sustained use of medicines. This journal is indexed on PubMed Central, CAS,

\section{Dovepress}

EMBase, Scopus and the Elsevier Bibliographic databases. The manuscript management system is completely online and includes a very quick and fair peer-review system, which is all easy to use. Visit http://www.dovepress.com/testimonials.php to read real quotes from published authors. 